\title{
Periodic Solutions for Two Dimensional Quartic Non-Autonomous Differential Equation
}

\author{
Saima Akram ${ }^{1, *}$, Allah Nawaz ${ }^{1}$, Muhammad Bilal Riaz ${ }^{2}$ and Mariam Rehman ${ }^{3}$ \\ ${ }^{1}$ Centre for Advanced Studies in Pure and Applied Mathematics, Bahauddin Zakariya University, Multan, 60000, Pakistan \\ ${ }^{2}$ Department of Mathematics, University of Management and Technology, Lahore, 54000, Pakistan \\ ${ }^{3}$ Department of Information Technology, Government College University, 38000, Faisalabad \\ *Corresponding Author: Saima Akram. Email: saimaakram@bzu.edu.pk \\ Received: 25 April 2021; Accepted: 15 June 2021
}

\begin{abstract}
In this article, the maximum possible numbers of periodic solutions for the quartic differential equation are calculated. In this regard, for the first time in the literature, we developed new formulae to determine the maximum number of periodic solutions greater than eight for the quartic equation. To obtain the maximum number of periodic solutions, we used a systematic procedure of bifurcation analysis. We used computer algebra Maple 18 to solve lengthy calculations that appeared in the formulae of focal values as integrations. The newly developed formulae were applied to a variety of polynomials with algebraic and homogeneous trigonometric coefficients of various degrees. We were able to validate our newly developed formulae by obtaining maximum multiplicity nine in the class $\mathrm{C}_{4,1}$ using algebraic coefficients. Whereas the maximum number of periodic solutions for the classes $\mathrm{C}_{4,4} ; \mathrm{C}_{5,1} ; \mathrm{C}_{5,5} ; \mathrm{C}_{6,1} ; \mathrm{C}_{6: 6} ; \mathrm{C}_{7,1}$ is eight. Additionally, the stability of limit cycles belonging to the aforementioned classes with algebraic coefficients is briefly discussed. Hence, we conclude from the above-stated facts that our new results are a credible, authentic and pleasant addition to the literature.
\end{abstract}

Keywords: Limit cycle; nonlinear equation; quartic differential equation; algebraic and trigonometric coefficients; focal values

\section{Introduction}

Most of the real-world problems in nature are multidimensional and when modeled, they arise as higherorder ordinary differential equations. We are interested in those models which are periodic and depend on time and are usually known as non-autonomous. This article contains several recent developments and advances in calculations of periodic solutions and their applications in various areas of the mathematical, physical and engineering sciences. We have investigated upper bounds for the non-autonomous ordinary differential equation (ODE) of the cubic degree [1-3]. The primary question striking in our minds is to investigate the maximum number of periodic solutions when the degree of non-autonomous (ODE) is increased from three to four; so, we started working for the quartic system. The analysis of periodic solutions is vital because they frequently arise as real-world problems from financial matters such as modelling economic processes to complex space robotics, from galaxies to weather forecasting models.

This work is licensed under a Creative Commons Attribution 4.0 International License, which permits unrestricted use, distribution, and reproduction in any medium, provided the original work is properly cited. 
Many physics problems are related to nonlinear analysis like relativity, elasticity, chaotic dynamics and Navier stokes equation in fluid dynamics. Bendixson's negative criterion, based on the connection between compound matrices and (ODE's), is applied to prove the nonexistence of periodic orbits and then nonexistence of Hopf bifurcation. Almost every bit of life needs this analysis, even inside the body as cardiac rhythms as blood flow, please see the example, [4-6]. The aesthetic appeal of periodic solutions may explain why so many people have become intrigued by these ideas. Alwash et al. $[7,8]$ developed the theoretical foundations of periodic solutions of differential equations.

Alwash et al. [8] examine equation of the form:

$\dot{y}=g(z) y^{3}+d(z) y^{2}+u(z) y$,

to find the upper bound for the number of limit cycles for such system (1) in accordance with the second part of Hilbert's sixteenth problem. The coefficients $g$; $d$; and $u$ are real-valued continuous functions, but the independent variable is complex. We are principally concerned about the multiplicity of $y=0$ as a periodic solution, i.e., solutions satisfying

$y(0)=y(\sigma)$,

for $\sigma \in \mathrm{R}$ and look for information about the number of periodic solutions. We refer the reader to [8] for more extra pieces of information, however right here we do not forget that the multiplicity of a solution $\phi(\mathrm{t})$ of Eq. (1) satisfying Eq. (2) is the multiplicity of $\phi(0)$ as a root of the holomorphic function $q: c \rightarrow y(t, 0, c)-c ; y\left(t, t_{1}, c\right)$ is the solution satisfies $y\left(t, t_{1}, c\right)=c$. Here we take a multiplicity of the periodic solution as ' $\boldsymbol{k}$ ' of the function $\phi(t)$. Rouche's theorem is used for the function $q$, it is assumed that we have at most $k$ periodic solutions in the regions nearby $\phi$ (counting multiplicity): see, for example, ([2], Theorem 2.4) when sufficiently small perturbation of the equation is in an account for the following quartic equation:

$\dot{y}=\varepsilon y^{4}+g(z) y^{3}+d(z) y^{2}+u(z) y$,

For sufficiently small perturbation $\varepsilon$, the quartic equation preserves the same number of periodic solutions as Eq. (1). We used scaling $y \rightarrow \varepsilon^{1 / 3} y$ to get the leading coefficient of Eq. (3) as one and this Eq. (3) becomes as:

$\dot{y}=y^{4}+\varepsilon^{-2 / 3}(z) y^{3}+\varepsilon^{-1 / 3}(z) y^{2}+u(z) y$,

To make the above equation simple, which is helpful for lengthy calculations that arise in numerical integration, we rewrite Eq. (4) as:

$\dot{y}=y^{4}+g(z) y^{3}+d(z) y^{2}+u(z)$,

We are mainly focused on finding the multiplicity of periodic solutions of $y=0$ greater than one for Eq. (1) as was done by researchers in [7], without loss of generality; we could take $u(z) \simeq 0$; as was taken in [1]. In this paper, we are considering the corresponding equation:

$\dot{y}=y^{4}+g(z) y^{3}+d(z) y^{2}$,

There is another reason for our interest in Eq. (5). We say this system to be non-autonomous, nonlinear because all the ' $y$ ' on the right-hand side appear to a power greater than one. Because of the non-autonomous equation, equilibrium states are not usually associated with Eq. (6). The general form of the above Eq. (6) is as follows: 
$\dot{y}=y^{n}+p_{1}(z) y^{n-1}+p_{2}(z) y^{n-2}+, \ldots,+p_{0}(z) ; \quad$ here $p_{0}(z)=1$.

Moreover, the coefficients were considered to be periodic functions, please see the example, [8-11]. However, the questions related to Hilbert's sixteenth problem are reduced to polynomial equations in which $p_{0}$ has zeros. They showed that for $n=3$, Eq. (7) has precisely three periodic solutions. Here, the results of [12] no longer hold; they have presented some examples in [13] which showed that there is no idea about the upper bound for the number of periodic solutions when $n \geq 4$ and $p_{0}(z)=1$; until some coefficients are restricted. Earlier, Shahshahani [14]; determined the multiplicity of Eq. (6) when $g(z), d(z)$ and $u(z)$ are polynomial functions of $\mathrm{z}$. We also present in Section 3 that the conjecture made in [14] is false.

In Section 2, some results from the literature are employed and formulae for calculating the maximum number of periodic solutions, greater than eight are formulated and presented. In Section 3, we calculated the periodic multiplicity for various classes of coefficients. In the last section, we present conclusions and discussions.

\section{Calculation of Multiplicity}

In this section, we recall some important concepts necessary to understand the presented method; for instance, see [1]. For the sake of multiplicity of the zero solution of Eq. (5), we observe that in a neighborhood of $y=0$, we can write

$y(z, 0, c)=\sum_{i=0}^{\infty} u_{i}(z) c^{i}$

for $0 \leq z \leq \sigma$; the functions $u_{i}(z)$ are continuous and $u_{1}(0)=1$ and $u_{i}(0)=0$ for $i>1$. The multiplicity $(\mu)$ is " $\mu=k$ " if

$$
\left.\begin{array}{c}
u_{1}(\sigma)=1 \\
u_{2}(\sigma)=u_{3}(\sigma)=\ldots=u_{i-1}(\sigma)=0 .
\end{array}\right\}
$$

However, $u_{i}(\sigma) \neq 0$. To calculate the functions $u_{i}(z)$, we placed the expression (8) into (5) and achieved a set of linear differential equations which can be solved recursively. We will study from Eq. (9) that $u_{1}(z)=u_{1}(z) v(z)$, where $u_{1}(z)$ is set as:

$u_{-} 1(z)=\mathrm{e}^{\int_{0}^{\sigma} v(\mathrm{~s}) \mathrm{ds}}$.

Hence, $y=0$ is a multiple solution iff $\int_{0}^{\sigma} v(\mathrm{~s})$ ds. Since we were right here interested in equations, wherein the multiplicity of the origin is greater than one, we recall that $\int_{\mathrm{o}}^{\sigma} v=0$. At that point, we apply the transformation $y \rightarrow y p^{n-1}$, wherein $p(z)=\mathrm{e}^{\int_{0}^{z} v}$ and obtain

$y=(p(z))^{3} y^{4}+(p(z))^{2} y^{3}+p(z) v(z) y^{2}$.

Since $p$ is periodic, the function $q$ is unchanged through the transformation and therefore the periodic solution of Eq. (5) and Eq. (10) have identical preliminary points and multiplicities. Additionally, the transformation of the independent prompts to the Eq. (6). For Eq. (6), the functions $u_{i}(z)$, for $i>1$ are calculated by the following relation: 
$\dot{u}_{i}=\left(\begin{array}{c}\sum_{j+k+l+m=i} \\ j, k, l, m \geq 1\end{array}\right)\left(u_{j} u_{k} u_{l} u_{m}\right)+\gamma(z)\left(\begin{array}{c}\sum_{j+k+l=i} \\ j, k, l \geq 1\end{array}\right)\left(u_{j} u_{k} u_{l}\right)+\delta(z)\left(\begin{array}{c}\sum_{j+k=i} \\ j, k \geq 1\end{array}\right)\left(u_{j} u_{k}\right)$.

With $u_{1}(z)=1$. For $i \leq 8$ functions $u_{i}(z)$ and $x_{i}$ are provided in [1], for $i=9$, we have calculated $u_{9}(z)$ and $\chi_{9}$; and present them below in Theorems 2.1 and 2.2. Some tremendously complicated computations are involved; hence, we omit some details and we write results.

In this article, we present a novel approach for finding periodic solutions. By using Theorem 2.2, we can find the highest periodic solutions as 9. We base this method on the construction of Akram et al. [1]. The approach can apply to other planar systems as well.

When we started working for quartic non-autonomous (ODE), for this investigation, formulae for finding upper bounds greater than 8 are not present in the literature up to now. We came up with many challenges while working with this problem of quartic type. The first task is to compute these new formulas, which are previously unavailable in the literature. By putting in many efforts, we succeeded in constructing the new formula $u_{9}$ and $\chi_{9}$, which are given in theorems 2.1 and 2.2. In the below theorem, we use the notation " $($.$) " for indefinite integral such as \bar{\delta}=\int \delta(s) d s$.

Theorem 2.1 For Eq. (6); the functions $\mathrm{u}_{2}, \mathrm{u}_{3}, \ldots, \mathrm{u}_{9}$ in the expansion (11) are as follows:

$$
\begin{aligned}
& u_{2}=\bar{\delta}, \\
& u_{3}=\bar{\delta}^{2}+\bar{\gamma}, \\
& u_{4}=\bar{\delta}^{3}+2 \bar{\delta} \bar{\gamma}+\bar{\delta} \bar{\gamma}+z \\
& u_{5}=\bar{\delta}^{4}+3 \bar{\delta}^{2} \bar{\gamma}+\bar{\delta}^{2} \gamma+3 \bar{\delta} \bar{\delta} \gamma+2 \overline{\bar{\delta}}+\frac{3}{2} \bar{\gamma}^{2}+2 z \bar{\delta}, \\
& u_{6}=\bar{\delta}^{5}+4 \bar{\delta}^{3} \bar{\gamma}+\overline{\bar{\delta}^{3} \gamma}+3 \bar{\delta}^{2} \overline{\bar{\delta} \gamma}+3 \bar{\delta}^{2}+4 \bar{\delta} \overline{\bar{\delta}}+2 \bar{\delta} \bar{\delta}^{2} \gamma-\frac{1}{2} \overline{\delta \bar{\gamma}^{2}}+\frac{9}{2} \bar{\delta} \bar{\gamma}^{2}+3 \overline{\bar{\delta} \gamma} \bar{\gamma}+\overline{\bar{\gamma}}+3 z\left(\bar{\delta}^{2}+\bar{\gamma}\right), \\
& u_{7}=\bar{\delta}^{6}+5 \bar{\delta}^{4} \bar{\gamma}+\overline{\bar{\delta}^{4} \gamma}+4 \bar{\delta}^{3} \overline{\bar{\delta} \gamma}+2 \bar{\delta} \overline{\bar{\delta}^{3} \gamma}+4 \overline{\bar{\delta}^{3}}+3 \bar{\delta}^{2} \overline{\bar{\delta}^{2} \gamma}+6 \bar{\delta} \bar{\delta}^{2}+6 \bar{\delta}^{2} \overline{\bar{\delta}}+\frac{27}{2} \bar{\delta}^{2} \bar{\gamma}^{2}+3 \overline{\bar{\delta}^{2} \gamma \bar{\gamma}} \\
& -\overline{\delta \bar{\gamma}^{2}} \bar{\delta}-2 \overline{\delta \bar{\delta} \bar{\gamma}^{2}}+6(\overline{\bar{\delta} \gamma})^{2}+2 \bar{\delta} \overline{\bar{\gamma}}+4 \overline{\bar{\delta} \bar{\gamma}}-4(\overline{\delta \bar{\gamma}})^{2}+6 \overline{\bar{\delta}} \bar{\gamma}+\frac{5}{2} \bar{\gamma}^{3}+4 z\left(\bar{\delta}^{3}+3 \overline{\bar{\delta} \gamma}+2 \overline{\delta \bar{\gamma}}\right)+2 z, \\
& u_{8}=\bar{\delta}^{7}+6 \bar{\delta}^{5} \bar{\gamma}+\overline{\bar{\delta}^{5} \gamma}+2 \bar{\delta} \overline{\bar{\delta}^{4} \gamma}+5 \bar{\delta}^{4} \overline{\bar{\delta} \gamma}+3 \bar{\delta}^{2} \overline{\bar{\delta}^{3} \gamma}+4 \bar{\delta}^{3} \overline{\bar{\delta}^{2} \gamma}+8 \bar{\delta} \overline{\bar{\delta}^{3}}+9 \bar{\delta}^{2} \overline{\bar{\delta}}^{2}-8 \bar{\delta}^{3} \overline{\bar{\delta}}+5 \bar{\delta}^{4} \\
& +\frac{75}{2} \bar{\delta}^{3} \bar{\gamma}^{2}+3 \overline{\bar{\delta}^{3} \gamma \bar{\gamma}}+12 \overline{\bar{\delta}^{2} \gamma} \bar{\delta} \bar{\gamma}-\overline{\bar{\delta}^{2} \overline{\delta \bar{\gamma} \gamma}}-6 \overline{\bar{\delta}^{2} \delta \bar{\gamma}^{2}}-25 \bar{\delta}^{2} \overline{\delta \bar{\gamma} \bar{\gamma}}-\frac{3}{2} \bar{\delta}^{2} \overline{\delta \bar{\gamma}^{2}}-4 \overline{\bar{\delta}^{2} \gamma} \overline{\delta \bar{\gamma}}+5 \bar{\delta}(\overline{\delta \bar{\gamma}})^{2}-4 \bar{\delta} \overline{\delta \bar{\delta} \bar{\gamma}^{2}} \\
& +9 \overline{\bar{\delta}^{2} \bar{\gamma}}-\overline{\overline{\bar{\delta}^{2}} \gamma}-2 \overline{\bar{\delta} \bar{\delta} \gamma}+9 \bar{\delta}^{2} \bar{\gamma}+3 \bar{\delta}^{2} \bar{\gamma}+16 \bar{\delta} \overline{\bar{\delta}} \bar{\gamma}+8 \bar{\delta} \overline{\bar{\delta}} \bar{\gamma}+10 \overline{\bar{\delta}} \overline{\bar{\delta} \gamma}-2 \overline{\bar{\delta}}-\frac{1}{2} \overline{\delta \bar{\gamma}^{3}}-\frac{3}{2} \overline{\delta \bar{\gamma}^{2}} \bar{\gamma}+10 \bar{\delta} \bar{\gamma}^{3} \\
& +\frac{15}{2} \overline{\bar{\delta}} \bar{\gamma}^{2}+\frac{3}{2} \overline{\bar{\gamma}^{2}}+3 \bar{\gamma} \bar{\gamma}+z\left(5 \bar{\delta}^{4}+15 \bar{\delta}^{2} \bar{\gamma}+5 \overline{\bar{\delta}^{2} \gamma}+10 \bar{\delta} \bar{\delta} \bar{\gamma}+10 \overline{\bar{\gamma}}+\frac{15}{2} \bar{\gamma}^{2}\right)+5 z^{2} \bar{\delta},
\end{aligned}
$$




$$
\begin{aligned}
& u_{9}=\bar{\delta}^{8}-\overline{\bar{\delta}^{3} \bar{\gamma}}+4 \overline{\overline{\bar{\delta}^{3}} \gamma}+58 \overline{\bar{\delta} \gamma} \bar{\delta}^{2}+12 \overline{\overline{\bar{\delta}^{2}}}+20(\overline{\bar{\delta}})^{2}+10 \overline{\overline{\bar{\delta}^{2}} \gamma \bar{\delta}}-2 \overline{\overline{\delta \bar{\gamma}^{2}}}+2 \overline{\bar{\delta} \bar{\gamma}^{2}}+24 \overline{\bar{\delta} \gamma \bar{\gamma}}-2 \overline{\bar{\gamma}}
\end{aligned}
$$

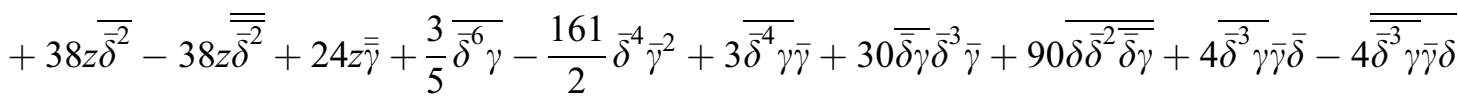

$$
\begin{aligned}
& +12 \overline{\bar{\delta}^{3}} \bar{\gamma}+11 \bar{\gamma} \bar{\delta}^{2} \overline{\bar{\delta}^{2} \gamma}+\frac{36}{3} \bar{\gamma} \bar{\delta} \bar{\delta}^{2}+34 \bar{\gamma} \bar{\delta}^{2} \overline{\bar{\delta}}+\frac{39}{2} \bar{\delta}^{2} \bar{\gamma} 3+\frac{15}{2} \bar{\gamma}^{2} \bar{\delta}^{2} \gamma+21 \bar{\gamma}(\bar{\delta} \gamma)^{2}-2 \bar{\gamma} \bar{\delta} \bar{\delta} \bar{\gamma}^{2}+4 \bar{\gamma} \bar{\gamma} \bar{\delta} \\
& +\frac{35}{8} \bar{\gamma}^{4}+12 \bar{\gamma} \overline{\bar{\delta}} \bar{\gamma}+15 \bar{\gamma}^{2} \overline{\bar{\delta}}+\frac{58}{3} \bar{\gamma} \bar{\delta}^{3} z-58 \bar{\gamma} \delta \bar{\delta}^{2} z+24 \bar{\gamma} \bar{\delta} \bar{\gamma}-24 \overline{\bar{\gamma}^{2} \delta}+9 \bar{\gamma} z^{2}-36 \overline{\gamma \bar{\gamma} \bar{\delta} \bar{\delta} \gamma} \\
& -66 \bar{\gamma} \delta \bar{\delta}^{2} z+42 \bar{\gamma} \bar{\delta} \gamma z-42 \overline{\bar{\gamma} \overline{\bar{\delta} \gamma}}-42 \bar{\gamma}^{2} \bar{\delta} z+42 \overline{\bar{\gamma}^{2} \delta z}+9 \bar{\gamma} \bar{\delta}^{3} \overline{\bar{\delta}^{2} \gamma}-9 \bar{\gamma} \bar{\delta}^{5} \gamma+27 \bar{\gamma} \delta \bar{\delta}^{2} \overline{\bar{\delta}^{2} \gamma} \\
& -12 \bar{\gamma}(\overline{\delta \bar{\gamma}})^{2}+24 \overline{\bar{\gamma}^{2} \delta \overline{\delta \bar{\gamma}}}-6 \overline{\gamma \bar{\delta} \bar{\delta} \bar{\gamma}^{2}}+2 \bar{\delta} \overline{\delta^{5} \gamma}+7 \bar{\gamma} \bar{\delta}^{6}+3 \bar{\delta}^{2} \overline{\bar{\delta}^{4} \gamma}+\frac{32}{5} \bar{\delta}^{5} \bar{\delta} \bar{\gamma}+4 \bar{\delta}^{3} \overline{\bar{\delta}^{3} \gamma}+5 \bar{\delta}^{4} \overline{\bar{\delta}^{2} \gamma} \\
& +12 \bar{\delta}^{3} \overline{\bar{\delta}}^{2}+12 \bar{\delta}^{2} \bar{\delta}^{3}+10 \bar{\delta}^{4} \overline{\bar{\delta}}+10 \bar{\delta} \bar{\delta}^{4}+\frac{101}{3} \bar{\delta}^{4} \bar{\gamma}+8 \bar{\gamma} \bar{\delta} \overline{\delta^{3} \gamma}+19 \bar{\gamma} \bar{\delta}^{2} \overline{\bar{\delta}^{2} \gamma}-2 \bar{\delta} \overline{\bar{\delta}^{2} \bar{\delta} \bar{\gamma} \gamma}+\frac{82}{3} \bar{\gamma} \overline{\bar{\delta}^{3} \overline{\delta \bar{\gamma}}} \\
& -12 \bar{\delta} \overline{\bar{\gamma}^{2} \delta \bar{\delta}^{2}}-\frac{52}{3} \bar{\gamma} \bar{\delta}^{3} \overline{\delta \bar{\gamma}}-2 \bar{\delta}^{3} \overline{\delta \bar{\gamma}^{2}}-8 \overline{\delta \overline{\bar{\delta}^{2} \gamma} \overline{\delta \bar{\gamma}}}+\bar{\delta}^{2}(\overline{\delta \bar{\gamma}})^{2}-6 \bar{\delta}^{2} \overline{\delta \bar{\delta} \bar{\gamma}^{2}}+18 \bar{\delta} \overline{\bar{\delta}^{2} \bar{\gamma}}-\overline{2 \delta} \overline{\overline{\bar{\delta}^{2} \gamma}} \\
& -4 \bar{\delta} \overline{\bar{\delta}} \bar{\delta} \gamma+12 \bar{\gamma} \bar{\delta} \overline{\delta^{2}}+4 \overline{\bar{\gamma}} \bar{\delta}^{3}-12 \bar{\gamma} \overline{\bar{\delta}} \bar{\delta}^{2}+12 \bar{\delta}^{2} \bar{\delta} \bar{\gamma}+10 \bar{\delta}^{2} \bar{\delta} \bar{\gamma}-10 \overline{\bar{\delta}^{3} \gamma}-4 \bar{\delta} \overline{\bar{\delta}}-\overline{\delta \bar{\gamma}^{3}} \bar{\delta}+2 \bar{\gamma} \bar{\delta} \overline{\delta \bar{\gamma}^{2}} \\
& +\frac{49}{2} \bar{\gamma}^{3} \bar{\delta}^{2}+24 \bar{\gamma}^{2} \bar{\delta} \bar{\delta} \gamma+3 \overline{\bar{\gamma}^{2}} \bar{\delta}+8 \bar{\gamma} \bar{\delta} \bar{\gamma}-20 \overline{\bar{\gamma} \bar{\delta}}+10 \bar{\delta}^{3} \bar{\gamma} \overline{\bar{\delta} \gamma}+4 \overline{\bar{\delta}} \bar{\delta} \bar{\delta} \bar{\gamma}-4 \overline{\bar{\delta} \bar{\delta}^{2} \gamma}+2 \overline{\bar{\delta} \gamma} \bar{\delta} \overline{\bar{\delta}^{2} \gamma} \\
& -2 \overline{\bar{\delta}^{2} \gamma \bar{\delta}^{2} \gamma}+\bar{\delta}^{5} z+\frac{56}{3} \bar{\gamma}^{3} z+12 \delta \bar{\delta}^{2} \gamma z+10 \bar{\delta}^{2} \bar{\delta} \gamma z+20 \overline{\bar{\gamma}} \bar{\delta} z-20 \overline{\bar{\gamma}} \bar{\delta} z+24 \bar{\gamma}^{2} \bar{\delta} z-9 \bar{\delta}^{2} \bar{\delta} \bar{\gamma} z \\
& +9 \overline{\bar{\delta}^{2} \overline{\delta \bar{\gamma}} z}+9 \bar{\delta}^{2}(\overline{\bar{\delta}})^{2} z+4 \overline{\bar{\delta}} \bar{\delta} z+9 \bar{\delta}^{2} z^{2} \text {. }
\end{aligned}
$$

By using the above mentioned functions of Theorem 2.1, we can obtain Theorem 2.2, under some suitable conditions.

Theorem 2.2 The solution $y=0$ of (6) has a multiplicity $\mathrm{k}$, wherever $2 \leq k \leq 9$ if and only if $x_{2}=x_{3}=, \ldots, x_{k-1}=0$ and $x_{k} \neq 0$ where

$$
\begin{aligned}
& x_{2}=\int_{0}^{\sigma} \delta, \\
& x_{3}=\int_{0}^{\sigma} \gamma, \\
& x_{4}=\int_{0}^{\sigma}(\gamma \bar{\delta}+1), \\
& x_{5}=\int_{0}^{\sigma}\left(\gamma \bar{\delta}^{2}+2 \bar{\delta}\right), \\
& x_{6}=\int_{0}^{\sigma}\left(2 \gamma \bar{\delta}^{3}+6 \bar{\delta}^{2}-\delta \bar{\gamma}^{2}+2 \bar{\gamma}\right), \\
& x_{7}=\int_{0}^{\sigma}\left(\gamma \bar{\delta}^{4}+4 \bar{\delta}^{3}-2 \delta \bar{\delta} \bar{\gamma}^{2}+4 \bar{\delta} \bar{\gamma}\right),
\end{aligned}
$$


$\varkappa_{8}=\int_{0}^{\sigma}\left(\gamma \bar{\delta}^{5}+5 \bar{\delta}^{4}-6 \bar{\delta}^{2}-\bar{\delta}^{2} \bar{\delta} \bar{\gamma} \gamma-\bar{\delta}^{2} \gamma+9 \bar{\delta}^{2} \bar{\gamma}-2 \gamma \overline{\bar{\delta}} \overline{\bar{\delta}}-\frac{1}{2} \bar{\gamma}^{3} \delta-2 \overline{\bar{\delta}}+\frac{3}{2} \bar{\gamma}^{2}\right)$

and

$$
\begin{aligned}
& x_{9}=\int_{0}^{\sigma}\left(\gamma \bar{\delta}^{6}+10 \bar{\delta}^{5}+\frac{95}{9} \bar{\gamma} \bar{\delta}^{3}+\frac{290}{3} \overline{\bar{\delta}} \bar{\delta}^{2}+20 \overline{\bar{\delta}^{2}}+\frac{50}{3} \bar{\delta} \overline{\delta^{2} \gamma}-\frac{10}{3} \overline{\delta \bar{\gamma}^{2}}+\frac{10}{3} \bar{\delta} \bar{\gamma}^{2}+40 \bar{\gamma} \bar{\delta} \bar{\gamma}-\frac{100}{3} \overline{\bar{\gamma}}\right. \\
& +150 \delta \bar{\delta}^{2} \bar{\delta} \bar{\gamma}-\frac{20}{3} \bar{\gamma} \delta \overline{\bar{\delta}^{3} \gamma}-60 \gamma \bar{\gamma} \bar{\delta} \bar{\delta} \gamma-70 \bar{\gamma} \bar{\delta} \gamma-15 \bar{\delta}^{5} \bar{\gamma} \gamma+40 \delta \bar{\gamma} \overline{\bar{\gamma}}+45 \delta \bar{\delta}^{2} \bar{\gamma} \overline{\bar{\delta}^{2} \gamma}-10 \gamma \overline{\delta \bar{\delta} \bar{\gamma}^{2}} \\
& +\frac{410}{9} \gamma \bar{\delta}^{3} \overline{\delta \bar{\gamma}}-\frac{40}{3} \delta \overline{\bar{\delta}^{2}} \gamma \bar{\delta} \bar{\gamma}-\frac{100}{3} \bar{\delta} \overline{\bar{\gamma}}+15 \bar{\delta}^{2} \overline{\delta \bar{\gamma}}+24 \delta \bar{\gamma}^{2} \bar{\delta} \bar{\gamma}-\frac{20}{3} \bar{\delta}^{2} \overline{\bar{\delta}} \gamma-\frac{100}{3} \gamma \bar{\delta}^{2} \overline{\bar{\delta}^{2} \gamma}+70 \delta \bar{\gamma}^{2} z \\
& \left.-\frac{620}{3} \bar{\gamma} \bar{\delta}^{2} \delta z-\frac{100}{3} \bar{\gamma} \bar{\delta} z\right)
\end{aligned}
$$

Assume that $\chi_{i}=u_{i}(\sigma)$, at that point $\mu=i$ if $\chi_{1}=1$ and $\chi_{k}=0$ for $2 \leq k \leq i-2$ but $\chi_{i} \neq 0$. These $x_{i}$ 's are known as focal values.

Proof By definition, the multiplicity of the zero solution is $\mathrm{k}$ if $a_{n}(\sigma)=0$ for $2 \leq n \leq k-1$ and $u_{k}(\sigma) \neq 0$. Write $\xi_{k}=u_{k}(\sigma)$ and let $\chi_{k}$ be the value of $\xi_{k}$ when $\xi_{n}=0$ for $n<k$.

Since $u_{2}(z)=\int_{\mathrm{o}}^{\sigma} \delta$, we have $\xi_{2}=\int_{\mathrm{o}}^{\sigma} \delta$. With $\xi_{2}=0$, we have $\xi_{3}=\int_{\mathrm{o}}^{\sigma} \gamma$ and $\xi_{4}=\int_{\mathrm{o}}^{\sigma}(\gamma \bar{\delta}+1)$; hence $\chi_{3}$ and $\chi_{4}$ as stated. Next, suppose that $\xi_{2}, \xi_{3}=0$ and substitute the relations $\int_{0}^{\sigma} \delta=\int_{0}^{\sigma} \gamma=0$ into the expressions for $u_{5}(\sigma)$ and $u_{6}(\sigma)$ given in theorem 2.1; we obtain $x_{4}=\int_{0}^{\sigma}(\gamma \bar{\delta}+1), x_{5}=\int_{0}^{\sigma}\left(\gamma \bar{\delta}^{2}+2 \bar{\delta}\right)$.

Finally, we suppose that $\xi_{4}=0$ (as well as $\xi_{2}=\xi_{3}=0$ ). We substitute the relations $\int_{\mathrm{o}}^{\sigma} \delta=\int_{\mathrm{o}}^{\sigma} \gamma=\int_{\mathrm{o}}^{\sigma}(\gamma \bar{\delta}+1)=0$ into the expressions for $u_{7}(\sigma)$ and $u_{8}(\sigma)$; then $\chi_{7}=\int_{\mathrm{o}}^{\sigma}\left(\gamma \bar{\delta}^{4}+4 \bar{\delta}^{3}\right.$ $\left.-2 \delta \bar{\delta} \bar{\gamma}^{2}+4 \bar{\delta} \bar{\gamma}\right)$

and $x_{8}=\int_{\mathrm{o}}^{\sigma}\left(\gamma \bar{\delta}^{5}+5 \bar{\delta}^{4}-6 \bar{\delta}^{2}-\bar{\delta}^{2} \bar{\delta} \bar{\gamma} \gamma-\overline{\bar{\delta}^{2} \gamma}+9 \bar{\delta}^{2} \bar{\gamma}-2 \gamma \overline{\bar{\delta}}-\frac{1}{2} \bar{\gamma}^{3} \delta-2 \overline{\bar{\delta}}-+\frac{3}{2} \bar{\gamma}^{2}\right)$.

Continuing in the same way we get: $x_{9}=\int_{0}^{\sigma}\left(\gamma \bar{\delta}^{6}+10 \frac{\bar{\delta}}{5}+\frac{95}{9} \bar{\gamma} \bar{\delta}^{3}+\frac{290}{3} \overline{\bar{\gamma}} \bar{\delta}^{2}+20 \bar{\delta}^{2}+\frac{50}{3} \bar{\delta} \bar{\delta}^{2} \gamma\right.$ $-\frac{10}{3} \overline{\delta \bar{\gamma}^{2}}+\frac{10}{3} \bar{\delta} \bar{\gamma}^{2}+40 \bar{\gamma} \bar{\delta} \bar{\gamma}-\frac{100}{3} \overline{\bar{\gamma}}+150 \delta \bar{\delta}^{2} \overline{\bar{\delta} \gamma}-\frac{20}{3} \bar{\gamma} \delta \bar{\delta}^{3} \gamma-60 \gamma \bar{\gamma} \bar{\delta} \bar{\delta} \gamma-70 \bar{\gamma} \bar{\delta} \bar{\gamma}-15 \bar{\delta}^{5} \bar{\gamma} \gamma+40 \delta \bar{\gamma} \bar{\delta} \bar{\gamma}$

$+45 \delta \bar{\delta}^{2} \bar{\gamma} \bar{\delta}^{2} \gamma-10 \gamma \overline{\delta \bar{\delta} \bar{\gamma}^{2}}+\frac{410}{9} \gamma \bar{\delta}^{3} \overline{\delta \bar{\gamma}}-\frac{40}{3} \delta \overline{\bar{\delta}^{2}} \gamma \bar{\delta} \bar{\gamma}-\frac{100}{3} \bar{\delta} \overline{\bar{\gamma}}+15 \bar{\delta}^{2} \overline{\delta \bar{\gamma}}+24 \delta \bar{\gamma}^{2} \overline{\delta \bar{\gamma}}-\frac{20}{3} \bar{\delta} \bar{\delta} \gamma-\frac{100}{3} \gamma \bar{\delta}^{2} \bar{\delta}^{2} \gamma$

$+70 \delta \bar{\gamma}^{2} z-\frac{620}{3} \bar{\gamma} \bar{\delta}^{2} \delta z-\frac{100}{3} \bar{\gamma} \bar{\delta} z$ ).

Now we are going to define the center and some necessary conditions for the center.

Definition An equilibrium point surrounded in its immediate neighborhood (not necessarily over the whole plane) by a closed path is called a center.

\section{Conditions for Center}

Corollary 2.1 If any $g(z)$ or $d(z)$ is identically zero and the other has a mean value of zero, then the origin is a center.

Corollary 2.2 For continuously odd differentiable functions $g(z)$ and $d(z)$ of period $\sigma$ : The origin is a center.

\section{Main Results}

We consider various classes in which two types of coefficients, namely $(z$ or $\cos (z)$ and $\sin (z))$ for $g(z)$ and $d(z)$ in Eq. (6), are considered for the calculations of periodic solutions. 


\subsection{Trigonometric Coefficients}

We have to consider Eq. (6), with coefficients in $\sin (z)$ and $\cos (z)$; here, the period is $\sigma=2 \pi$. We now go off to various classes of Eq. (6). For this, we make use of theorem 2.2; to verify the calculations in this module. The related computations are complicated and of a bulk extent. This symbolic representation like $\int_{0}^{2 \pi} z \cos ^{m}(z) \sin ^{n}(z) d z$ was overcome using computer algebra programming Maple18.

Lemma 3.1 If possibly $g(z)$ and $d(z)$ are polynomials in $\sin (z)$ and $\cos (z)$, all of whose terms are of odd degree. Then multiplicity is $\mu=4$ or $\mu>6$.

The above lemma is from Alwash et al. [7], but after solving theorems in the next section, we have concluded that this is also valid if $g(z)$ and $d(z)$ are of even degree, given in the following lemma.

Lemma 3.2 If $g(z)$ and $d(z)$ are polynomials in $\sin (z)$ and $\cos (z)$; all of whose terms are of even degree. Then multiplicity is $\mu=4$ or $\mu>6$.

Which are illustrated in the following theorems, throughout this paper, we use the symbol " $\mathrm{C}$ " to represent the class and we show the maximum multiplicity for classes with $\mu_{\max }$.

Homogeneous Polynomial for Classes $C_{4,4}, C_{5,5}$ and $C_{6,6}$

Theorem 3.1 Consider the class $\mathrm{C}_{4,4}$ for $\mathrm{Eq}$. (6). If the coefficients are:

$\gamma(z)=a_{\cos ^{4}}(z)+b \cos ^{3}(z) \sin (z)+\operatorname{csin}^{4}(z)+f \cos ^{2}(z) \sin ^{2}(z) ;$

$\delta(z)=d \cos ^{4}(z)+\operatorname{esin}^{4}(z)$.

Then we calculate $\mu_{\max }\left(C_{4,4}\right) \geq 8$.

Proof By using Theorem 2.2, we now calculate $\varkappa_{2}=\frac{3}{4} \pi(d+e)$,

$\chi_{3}=\frac{\pi(3 a+3 c+f)}{4}$.

From $\varkappa_{2}, \varkappa_{3}=0$, we substitute $d=-e, f=-3 a-3 c$ and calculate $\chi_{4}=\frac{\pi(16+b e)}{8}$. From $\chi_{4}=0$, by using the value of " $b$ " in terms of " $e$ " we calculate $x_{5}$ as $x_{5}=-\frac{\pi e^{2}(a+c)}{16}$.

If $\chi_{5}=0$ then, either $e=0$ or:

$a+c=0$.

If $e=0$ then $\delta(z)=0$ and for $\chi_{3}=0$, we can say from corollary 2.1, that the origin is a center. Utilizing (11) we put $a=-c$ and get

$\chi_{6}=\frac{\pi\left(3 e^{3}-4\right)}{4 e}$.

with $\chi_{7}$ as: $\chi_{7}=-\frac{2 c \pi}{9}$. For $\chi_{7}=0$, we substitute values of "c" and calculate $\chi_{8}$ as:

$\varkappa_{8}=\left(\frac{1538563}{419904}\right) \pi$.

Hence our conclusion is $\mu_{\max }\left(C_{4,4}\right) \geq 8$.

Theorem 3.2 Let the class $C_{5,5}$ for Eq. (6); with coefficients as follows:

$\gamma(z)=\operatorname{asin}^{5}(z)+b \cos ^{3}(z) \sin ^{2}(z)+c \cos ^{4}(z) \sin (z)+d \cos ^{5}(z)$, 
$\delta(z)=\operatorname{esin}^{5}(z)$

Then $\mu_{\max }\left(C_{5,5}\right) \geq 8$ is presented.

Proof It is easily calculated that $\chi_{2}=\chi_{3}=0$ and:

$\varkappa_{4}=-\frac{\pi(161 e b+689 e d-3840)}{1920}$.

If $\chi_{4}=0$, then, as $\pi \neq 0$, we substitute $d=\frac{3840}{689 e}-\frac{161}{689} b$ and calculate $\chi_{5}=0$ with $\chi_{6}$ as:

$\chi_{6}=\frac{e^{2} \pi(7576305 e b+2183801728)}{1693286400}$.

If $\chi_{6}=0$, then for $e=0$, gives that origin is the center. Thus we put $b=-\left(\frac{2183801728}{7576305 e}\right)$ and get $\chi_{7}=\left(\frac{64434587}{363662640}\right) e c \pi$.

Now, $\chi_{7}=0$ gives $c=0$, because $e \pi \neq 0$ and $\chi_{8}$ is as follows:

$\varkappa_{8}=\frac{\pi \varpi}{10687682598305969912832000 e^{2}}$.

where $\varpi=3463030403249893347931717632+1080907356115092660813824 e^{4} a$

$+455749311538699264881025 e^{6}$.

Hence, $\mu_{\max }\left(C_{5,5}\right) \geq 8$ is calculated.

Theorem 3.3 Consider class $\mathrm{C}_{6,6}$ for Eq. (6), with coefficients as follows:

$\gamma(z)=\operatorname{asin}^{6}(z)+\cos ^{5}(z) \sin (z)+d \cos ^{6}(z)$,

$\delta(z)=e \cos ^{6}(z)+f \sin ^{4}(z) \cos ^{2}(z)$.

Then $\mu_{\max }\left(C_{6,6}\right) \geq 8$.

Proof From theorem 2.2, we calculated $\chi_{2}=\frac{\pi(f+5 e)}{8}, \chi_{3}=\frac{5 \pi(a+d)}{8}$. From $\chi_{2}, \chi_{3}=0$, we substitute $a=-d, f=-5 e$ and calculate $\chi_{4}=\frac{\pi(1536+49 c e)}{768}$ and from $\varkappa_{4}=0$, by using the value of "c" in terms of "e" we calculate $x_{5}$ as:

$x_{5}=\left(\frac{65}{2048}\right) d e^{2}$

If $\chi_{5}=0$ then, as $e \neq 0$, so we put $d=0$ and get $\chi_{6}=\frac{\pi\left(-5529600+443401 e^{3}\right)}{1843968 e}$.

Further, for $\chi_{6}=0$ we put $=\left(\frac{5529600}{443401}\right)^{\frac{1}{3}}$, which results $\chi_{7}=0$ and $\chi_{8}$ as follows:

$\chi_{8}=\frac{1032896577437435266601938594527152689}{134056056318983503541054008197120000} \pi$.

Here it is a nonzero constant number. So, $\mu_{\max }\left(C_{6,6}\right) \geq 8$.

Now, for the polynomial z; we will explain the calculations of the maximum possible periodic solution for many classes of the Eq. (6).

\subsection{Polynomial Coefficient}

Let $C_{\mathrm{i}, \mathrm{j}}$ denotes the classes for the Eq. (6), where $i, j$ denotes the degree for $\gamma(\mathrm{z})$ and $\delta(\mathrm{z})$ accordingly. Using polynomial coefficient " $z$ " for various higher-order classes, we have calculated the possible 
maximum number of periodic solutions 9 for class $C_{4,1}$ and 8 for the classes $C_{5,1}, C_{6,1}, C_{7,1}$. The verification of the presented below theorems stems from papers by Alwash et al. $[7,8]$ and by Saima et al. please see the example, [1-3].

Remark 1. In the perturbation strategies defined in [1], the whole complement of $(j-2)$ real periodic solutions fails to yield; if it occurs, then there is $j<k$ such that $x_{j}=0$ whenever $x_{j-1} \neq 0$. This occurs while the multiplicity is necessarily odd. However, for the number of real periodic solutions, we will say from the "exchange of stability" argument that, If multiplicity $\mu$ is even, the origin is stable for $\chi_{\mu}<0$ and unstable if $x_{\mu}>0$. If $\mu$ is odd, then the origin is stable on the right and unstable on the left if $x_{\mu}<0$; while it is stable on the left and unstable on the right if $\chi_{\mu}>0$.

Theorem 3.4 Consider $C_{\mathrm{i}, 1}$ be the class of equations of the form (6) having degree i for $\gamma(z)$ and 1 for $\delta(z)$, where $i=4,5,6,7$. Then we conclude the results $\mu_{\max }\left(C_{i, 1}\right) \geq 8$, for $i=5,6,7$ and $\mu_{\max }\left(C_{4,1}\right) \geq 9$ accordingly.

Proof Firstly, we start for the degree of $\gamma(z)$ as 7 , i.e., $C_{7,1}$. The remaining classes having a degree less than 7 for $\gamma(z)$ are the special cases. Let

$$
\left.\begin{array}{c}
\gamma(z)=a+b z+c z^{2}+d z^{3}+e z^{4}+f z^{5}+g z^{6}+h z^{7} \\
\delta(z)=m+q z .
\end{array}\right\}
$$

1). For the class $C_{7,1}$, we restrict coefficients and put $e, f, g=0$, in (21). By using Theorem 2.2, we calculate $x_{2}=m+\frac{q}{2}$ and $\varkappa_{3}=a+\frac{b}{2}+\frac{c}{3}+\frac{d}{4}+\frac{h}{8}$.

Thus multiplicity of $z=0$ is $\mu=2$ if $\chi_{2} \neq 0$. And multiplicity is $\mu=3$ if $x_{2}=0$ but $x_{3} \neq 0$. If suppose $x_{2}, x_{3}=0$, then $x_{4}$ is given as:

$\varkappa_{4}=1+\frac{7}{1440} h q+\frac{1}{240} d q+\frac{1}{360} c q$.

If $x_{4}=0$ then by substituting

$h=-\frac{1440}{7 q}-\frac{6}{7} d-\frac{4}{7} c$,

we calculate $\chi_{5}=-\frac{q(3360+3 d q+2 c q)}{110880}$.

Now for $\varkappa_{5}=0$, either $q=0$ or $3360+3 d q+2 c q=0$. If $q=0$ then $\delta(z)=0$. Also $\varkappa_{3}=0$ gives, the mean value $\gamma(z)=0$. From corollary 2.1, origin is the center. Substituting value of $d$ as: $d=-\frac{3360}{3 q}-\frac{2}{3} c$. We obtain $\varkappa_{6}=-\frac{4\left(374 c q-782880-1683 q^{3}\right)}{1461915 q}$. If $\varkappa_{6}=0$, recalling that $q \neq 0$, we take

$c=\frac{1683}{374} q^{2}+\frac{782880}{374}\left(\frac{1}{q}\right)$.

Using Eq. (24), we calculate $x_{7}$ as:

$\varkappa_{7}=\left(\frac{88}{28665}\right) b q+\left(\frac{7487}{1261260}\right) q^{3}+\left(\frac{219888}{273581}\right)$.

If $\chi_{7}=0$, substituting the value of ' $\mathrm{b}$ ' in term of ' $\mathrm{q}$ ' and calculate towards $\chi_{8}$ as:

$\chi_{8}=-\frac{837814915675132032 q^{3}+143990207791969 q^{6}-99990153502176337920}{5030608785872237568 q^{2}}$.

For $\varkappa_{8}=0$, we can't proceed further. Therefore, $\mu_{\max }\left(C_{7,1}\right) \geq 8$. 
As the outcome of obtained multiplicity is even having the negative sign. So, by using remark 1, it can be concluded that the origin is stable.

2). Now, for the class $C_{6,1}$, we put $e, f, h=0$, in Eq. (21). By using theorem 2.2, we calculate

$\varkappa_{2}=m+\frac{q}{2}$,

and

$\chi_{3}=a+\frac{b}{2}+\frac{c}{3}+\frac{d}{4}+\frac{g}{7}$.

If suppose $\chi_{2}, \chi_{3}=0$, we calculate $\chi_{4}$ as:

$\varkappa_{4}=1+\frac{5}{1008} g q+\frac{1}{240} d q+\frac{1}{360} c q$.

If $x_{4}=0$ then by substituting

$g=-\frac{1008}{5 q}-\frac{21}{25} d-\frac{14}{25} c$

we calculate

$\chi_{5}=-\frac{q(13160+9 d q+6 c q)}{462000}$.

Now for $\chi_{5}=0$, either $q=0$ or $13160+9 d q+6 c q=0$. If $q=0$ then $\delta(z)=0$. Also, $\chi_{3}=0$ gives the mean value of $\gamma(z)=0$. From corollary 2.1, the origin is the center. By putting the value of ' $\mathrm{d}$ ' we obtain $\chi_{6}$ as:

$\chi_{6}=-\frac{2694 c q-12123 q^{3}-7085960}{2653560 q}$.

If $x_{6}=0$, recalling that $q \neq 0$, we take

$c=\frac{7085960}{2694 q}+\frac{12123}{2694} q^{2}$.

Using Eq. (26), we calculate $x_{7}$ as:

$\varkappa_{7}=\frac{449}{147420} b q+\frac{2729}{463320} q^{3}+\frac{105211651}{88413039}$.

If $x_{7}=0$, substituting the value of ' $b$ ' in term of ' $q$ ' we proceed towards $x_{8}$ as

$\chi_{8}=-\frac{733717712507810077572 q^{3}-87991271201849989542520+125212920801745293 q^{6}}{4404674611152546114384 q^{2}}$.

Now, we cannot proceed further with more calculations. So, concluded that multiplicity is 8 , i.e., $\mu_{\max }\left(C_{6,1}\right) \geq 8$. Using remark 1 , it can be concluded that the origin is stable due to its even and negative behaviour.

3). For the class $C_{5,1}$, we suppose that $g, h=0$ in Eq. (21). So

$\gamma(z)=a+b z+c z^{2}+d z^{3}+e z^{4}+f z^{5}$ and $\delta(z)=m+q z$. 
IASC, 2022, vol.31, no.3

Then by using theorem 2.2, we calculate

$\varkappa_{2}=m+\frac{q}{2}$,

$\chi_{3}=a+\frac{b}{2}+\frac{c}{3}+\frac{d}{4}+\frac{e}{5}+\frac{f}{6}$.

If $x_{2}, x_{3}=0$ then

$a=-\frac{b}{2}-\frac{c}{3}-\frac{d}{4}-\frac{e}{5}-\frac{f}{6}$,

$m=-\frac{q}{2}$.

Substituting these Eqs. (27) and (28), we have

$\gamma(z)=b\left(z-\frac{1}{2}\right)+c\left(z^{2}-\frac{1}{3}\right)+d\left(z^{3}-\frac{1}{4}\right)+e\left(z^{4}-\frac{1}{5}\right)+f\left(z^{5}-\frac{1}{6}\right)$,

$\delta(z)=q\left(z-\frac{1}{2}\right)$

Also we calculate $\chi_{4}$ as:

$\varkappa_{4}=1+\frac{5}{1008} f q+\frac{1}{210} e q+\frac{1}{240} d q+\frac{1}{360} c q$.

If $x_{4}=0$ then we put value of ' $e$ '

$e=-\frac{5040}{24 q}-\frac{21}{24} d-\frac{14}{24} c-\frac{25}{24} f$

and calculate $\chi_{5}$ as:

$\varkappa_{5}=-\frac{q(-5 f q+15120+3 d q+2 c q)}{604800}$.

If $\chi_{5}=0$, either $q=0$ or

$f=\frac{324}{q}+\frac{3}{5} d+\frac{2}{5} c$.

For $q=0$, from (29) $\delta(z)=0$. Also $x_{3}=0$ gives that the mean value $\gamma(z)=0$. Using corollary 2.1, origin is the center. By using $q \neq 0$ and (31), we have

$x_{6}=-\frac{64350 b q+37300 c q+10632 d q-11918700-39429 q^{3}}{2882880 q}$.

Now if we take $x_{6}=0$, then we put

$b=\frac{11918700}{64350 q}-\frac{37300}{64350} c-\frac{10632}{64350} d+\frac{39429}{64350} q^{2}$. 
And we calculate $\chi_{7}$ as

$\chi_{7}=-\frac{827}{1829520} c q+\frac{1060650323}{797127581250} d q-\frac{541753}{5285280}+\frac{93081}{22022000} q^{3}$.

If $\chi_{7}=0$, by using value of ' $c$ ' in terms of ' $d$ ' and ' $q$ ', we find $\chi_{8}$ as;

$$
x_{8}=-\frac{\begin{array}{c}
56105028902377434984806250 d q+10604160879626306419163625 d q^{4}+ \\
222680648946688729262610989 d^{2} q^{2}+14971274761300360631591290625 q^{3}+ \\
383832595451553004634864625 q^{6}-149276483225872273284531250
\end{array}}{10370649116108410151022000000000 q^{2}} .
$$

As $q \neq 0$ is considered above, we cannot calculate further. So, it is concluded that $\mu_{\max }\left(C_{5,1}\right) \geq 8$. This $x_{8}$ is even and its value has negative behavior. By using remark 1, it can be concluded that the origin is stable.

4). Let

$\gamma(z)=a+b z+c z^{2}+d z^{3}+e z^{4}$,

and

$\delta(z)=m+q z$.

Utilizing theorem 2.2, we get

$\varkappa_{2}=m+\frac{q}{2}$,

$\varkappa_{3}=a+\frac{b}{2}+\frac{c}{3}+\frac{d}{4}+\frac{e}{5}$.

Thus multiplicity of $z=0$ is $\mu=2$ if $x_{2} \neq 0$. And multiplicity $\mu=3$ if $x_{2}=0$ but $x_{3} \neq 0$. If $x_{2}, x_{3}=0$, then we calculate $x_{4}$ as:

$? \$ 4=1+\frac{1}{210} e q+\frac{1}{240} d q+\frac{1}{360} c q$.

If $\chi_{4}=0$, we substitute

$e=-\frac{5040}{24 q}-\frac{21}{24} d-\frac{14}{24}$.

By using Eq. (33) we compute $\chi_{5}=-\frac{q(15120+3 d q+2 c q)}{604800}$. If $\chi_{5}=0$, either $q=0$ or

$d=-\frac{15120}{3 q}-\frac{2}{3} c$.

If $q=0$ then $\delta(z)=0$. And $\chi_{2}=0$, gives that mean value of $\gamma(z)$ is zero. So by corollary 2.1, origin is the center. So $q \neq 0$, if Eq. (34) holds then $\chi_{6}=-\frac{q\left(2 c q-9 q^{3}-10080\right)}{1980}$.

If $x_{6}=0$ then recalling that $q \neq 0$, we put $c=\frac{5040}{q}+\frac{9}{2} q^{2}$ 
Moreover, $\chi_{7}$ takes form as:

$\varkappa_{7}=\frac{1}{330} b q+\frac{8}{1365} q^{3}+\frac{28}{11}$.

Now, if $\varkappa_{7}=0$, then

$b=-\frac{840}{q}-\frac{176}{91} q^{2}$.

Withholding Eq. (36), we have

$\varkappa_{8}=\frac{313021800+9409 q^{6}}{8198190 q^{2}}$.

If $x_{8}=0$, then as $q \neq 0$, so only possible substitution for the value of ' $q$ ' is $q=\left(-\frac{313021800}{9409}\right)^{1 / 6}$ and calculate $\chi_{9}$ as follows:

$\chi_{9}=\frac{193005492316097270013660400668166824453730263568721875}{50158432950253127263308993705898641}$.

This $x_{9}$ is a non-zero constant number. It is concluded that the class $C_{4,1}$ has nine periodic solutions, i.e., $\mu_{\max }\left(C_{4,1}\right) \geq 9$.

The multiplicity of this class is the highest one up to date. The outcome of maximum multiplicity for $\chi_{9}$ is odd and the sign assigned with it is positive. Using remark 1, it can be concluded that the origin is unstable on the right and stable on the left.

\subsection{Perturbation Method}

After calculating the periodic solutions, we will make a series of perturbations of the coefficients resulting from the periodic solution to bifurcate out of origin. In Hopf bifurcation, the creation of limit cycles near a fixed point is described. As the bifurcation parameter approaches some critical value, the limit cycle approaches the fixed point and the amplitude of the limit cycle approaches zero. The presented below method is followed by Alwash et al. [8] and Saima et al., please see the example, [3-5].

Theorem 3.6 For the class $C_{4,1}$; suppose that

$$
\gamma(z)=a+b z+c z^{2}+d z^{3}+e z^{4} \text { and } \delta(z)=m+q z,
$$

where

$$
\begin{aligned}
& q=\left(-\frac{313021800}{9409}\right)^{1 / 6}+\varepsilon_{1}, \\
& m=\left(-\frac{313021800}{9409}\right)^{1 / 6}-\left(\frac{1}{2}\right) \varepsilon_{1}+\varepsilon_{7}, \\
& b=-\left(\frac{(840)}{\left(\left(-\frac{313021800}{9409}\right)^{1 / 6}+\varepsilon_{1}\right)}\right)-\left(\frac{176}{91}\right)\left(\left(-\frac{313021800}{9409}\right)^{1 / 6}+\varepsilon_{1}\right)^{2}+\varepsilon_{2}, \\
& c=\left(\frac{(5040)}{\left(-\frac{313021800}{9409}\right)^{1 / 6}+\varepsilon_{1}}\right)+\left(\left(-\frac{313021800}{9409}\right)^{1 / 6}+\varepsilon_{1}\right)^{2}+\varepsilon_{3},
\end{aligned}
$$




$$
\begin{aligned}
& e=-\left(\frac{3150}{\left(-\frac{313021800}{9409}\right)^{1 / 6}+\varepsilon_{1}}\right)-\left(\frac{7}{8}\right) d-\left(\frac{21}{8}\right)\left(\left(-\frac{313021800}{9409}\right)^{1 / 6}+\varepsilon_{1}\right)^{2}-\left(\frac{7}{12}\right) \varepsilon_{3}+\varepsilon_{4}, \\
& d=-\left(\frac{8400}{\left(-\frac{313021800}{9409}\right)^{1 / 6}+\varepsilon_{1}}\right)-\left(\left(-\frac{313021800}{9409}\right)^{1 / 6}+\varepsilon_{1}\right)^{2}-\left(\frac{2}{3}\right) \varepsilon_{3}+\varepsilon_{5},
\end{aligned}
$$

and $a=-b z-c z^{2}-d z^{3}-e z^{4}+\varepsilon_{6}$.

Then seven real non-trivial periodic solutions exists, if $\varepsilon_{p}$ is small as compared to $\varepsilon_{p-1}, \forall p \leq 7$.

Theorem 3.7 For Eq. (6), with:

$$
\begin{aligned}
& \gamma(z)=-\frac{80330459}{1397630 q}+\frac{37282651}{69881500} q^{2}+\frac{901656327079}{1141514302500} d+\frac{578}{6435} \varepsilon_{1}-\frac{1}{2} \varepsilon_{2}+\frac{1}{24} \varepsilon_{3}-\frac{1}{5} \varepsilon_{4}+\varepsilon_{6}+ \\
& \left(\frac{44256805}{139763 q}-\frac{1343747}{279526} q^{2}-\frac{1546741671872}{285378575625} d-\frac{746}{1287} \varepsilon_{1}+\varepsilon_{2}\right) z+\left(-\frac{4875777}{21502 q}+\frac{2513187}{268775} q^{2}+\right. \\
& \left.\frac{2513187}{268775} d+\varepsilon_{1}\right) z^{2}+d z^{3}+\left(-\frac{3448284}{10751 q}-\frac{5832699}{537550} d-\frac{2513187}{268775} q^{2}-\varepsilon_{1}-\frac{25}{24} \varepsilon_{3}+\varepsilon_{4}\right) z^{4}+ \\
& \left(\frac{12540843}{53755 q}+\frac{5832699}{1343875} d+\frac{5026374}{1343875} q^{2}+\frac{2}{5} \varepsilon_{1}+\varepsilon_{3}\right) z^{5},
\end{aligned}
$$

and

$\delta(z)=-\frac{1}{2} q+\varepsilon_{6}+q z$.

If $\varepsilon_{p}(1 \leq p \leq 6)$, are taken to be non-zero and also $\left|\varepsilon_{6}\right| \leq\left|\varepsilon_{5}\right| \leq, \ldots, \leq\left|\varepsilon_{1}\right|$. Then there exist six distinct non-trivial real periodic solutions.

Theorem 3.8 For the Eq. (6), consider that

$$
\begin{aligned}
& \gamma(z)=-\frac{97077325}{3009391 q}+\frac{1679}{7744} q^{2}-\frac{1}{2} \varepsilon_{1}-\frac{1}{6} \varepsilon_{2}-\frac{1}{7} \varepsilon_{3}-\frac{1}{8} \varepsilon_{4}+\varepsilon_{6}+\left(-\frac{112555170}{429913 q}-\frac{7487}{3872} q^{2}+\varepsilon_{1}\right) z+ \\
& \left.\left(\frac{9}{2} q^{2}+\frac{391440}{187 q}+\varepsilon_{2}\right) z^{2}+\left(-\frac{470400}{187 q}-3 q^{2}-\frac{2}{3} \varepsilon_{2}+\varepsilon_{3}\right) z^{3}+\frac{5280}{7 q}-\frac{6}{7} \varepsilon_{3}+\varepsilon_{4}\right) z^{7}, \\
& \delta(z)=-\frac{1}{2} q+q z+\varepsilon_{5} .
\end{aligned}
$$

If $\varepsilon_{p}(1 \leq p \leq 6)$, are taken to be non-zero and also $\left|\varepsilon_{6}\right| \leq\left|\varepsilon_{5}\right| \leq, \ldots, \leq\left|\varepsilon_{1}\right|$. Then, it has six distinct non-zero, non-trivial real periodic solutions.

Proof The proof of theorems 3.5, 3.6 and 3.7 are similar as in ([3], theorem 4.2). Therefore, it is omitted. Finally, the concluding remarks of the article are described below. 


\section{Conclusion}

In this article, we calculate the possible maximum number of periodic solutions of the quartic differential equation. Earlier in the literature, there are no formulae available to calculate maximum multiplicity greater than 8 . Therefore, we calculated the formulae $u_{9}$ and $\chi_{9}$ given in Section 2 . By using them we can calculate multiplicity greater than eight. The second task is to check the authenticity and validity of these formulae. So, by using them, we succeeded in calculating the maximum number of periodic solutions 8 for classes $\mathrm{C}_{4,4} ; \mathrm{C}_{5,5} ; \mathrm{C}_{6,6} ; \mathrm{C}_{7,1} ; \mathrm{C}_{6,1} ; \mathrm{C}_{5,1}$; where we consider homogenous trigonometric and algebraic coefficients for the sake of variety regarding different polynomials. The most challenging task is to get the multiplicity of any class greater than eight, which assures us that the newly developed formula $\chi_{9}$ works as desired. We get multiplicity nine for the polynomial class $\mathrm{C}_{4,1}$. In this way, we develop new formulae and validate them through the use of previous literature. As future work, one can develop formula $\chi_{10}$ for calculating multiplicity greater than nine concerning quartic equation.

Acknowledgement: Methodology, A. N. and S. A.; writing-original draft preparation, S. A., A. N., M. B. R. and M. R.; formal analysis, S. A., A. N., M. R. and M. B. R.; writing-review and editing, S. A., M. R., M. B. R. and A. N.

Funding Statement: The authors received no specific funding for this study.

Conflicts of Interest: The authors declare that they have no conflicts of interest to report regarding the present study.

\section{References}

[1] S. Akram, A. Nawaz, N. Yasmin, H. Kalsoom and Y. M. Chu, "Periodic solutions for first order cubic nonautonomous differential equation with bifurcation analysis," Journal of Taibah University for Science, vol. 14, no. 1, pp. 1208-1217, 2020.

[2] S. Akram, A. Nawaz, H. Kalsoom, M. Idrees and Y. M. Chu, "Existence of multiple periodic solutions for cubic non-autonomous differential equation," Mathematical Problems in Engineering, vol. 2020, no. 1, pp. 1-14, 2020.

[3] S. Akram, A. Nawaz, T. Abdeljawad, A. Ghaffar and K. S. Nisar, "Calculation of focal values for first-order nonautonomous equation with algebraic and trigonometric coefficients," Open Physics, vol. 18, no. 1, pp. 738-750, 2020.

[4] S. Kumar, R. Kumar and R. Agarwal, "A study of fractional Lotka-Volterra population model using Haar wavelet and Adams-Bashforth-Moulton methods," Mathematical Methods in the Applied Sciences, vol. 43, no. 8, pp. 5564-5578, 2020.

[5] G. Nallappan, S. Sabarathinam, Z. Guisheng and Y. Qiang, "Dynamical analysis and sampled-data stabilization of memristor-based chua's circuits," IEEE Access, vol. 9, pp. 25648-25658, 2021.

[6] B. Unyong, V. Govindan, S. Bowmiya, G. Rajchakit and G. Nallappan, "Generalized linear differential equation using Hyers-Ulam stability approach,” AIMS Mathematics, vol. 6, no. 2, pp. 1607-1623, 2021.

[7] M. A. M. Alwash and N. G. Lloyd, "Periodic solutions of a quartic non-autonomous equation, Nonlinear analysis theory methods and applications," Nonlinear Analysis Theory Methods and Applications, vol. 11, no. 7, pp. 809820, 1987.

[8] M. A. M. Alwash and N. G. Lloyd, "Non-autonomous equation related to polynomial two-dimensional system," Proceedings of Royal Society Edinburgh, vol. 5, no. 1, pp. 129-152, 1987.

[9] S. Akram, A. Nawaz, N. Yasmin, A. Ghaffar and D. Baleanu, "Periodic solutions of some classes of one dimensional non-autonomous system," Frontiers in Physics, vol. 8, no. 264, pp. 1-14, 2020.

[10] N. G. Lloyd, "The number of periodic solutions of the equation $\mathrm{z}=\mathrm{z}^{\mathrm{n}}+\mathrm{p}_{1}(\mathrm{t}) \mathrm{z}^{\mathrm{n}-1}+\mathrm{p}_{2}(\mathrm{t}) \mathrm{z}^{\mathrm{n}-2}+, \ldots,+\mathrm{p}_{0}(\mathrm{z})$," Proceedings of London Mathematical Society, vol. 27, no. 3, pp. 667-700, 1973. 
[11] N. G. Lloyd, "Limit cycles of certain polynomial systems, nonlinear functional analysis and its applications," in S.P. Singh NATO ASI Series. Springer, Dordrecht, vol. 173, pp. 317-326, 1986.

[12] L. Neto, "On the number of solutions of the equations $d x / d z=\sum_{j=0}^{n} a_{j}(z) z, 0 \leq z \leq 1$ for which $x(0)=x(1)$," Inventiones Mathematicae, vol. 59, no. 6, pp. 67-76, 1980.

[13] N. G. Lloyd, "Small amplitude limit cycles of polynomial differential equations in ordinary differential equations and operators," Lecture Notes in Mathematics, Dordrecht: Springer, vol. 1032, pp. 346-357, 1982.

[14] S. Shahshahani, "Periodic solutions of polynomial first order differential equations," Nonlinear Analysis, vol. 5, no. 2, pp. 157-165, 1981. 